\title{
Acetyl-L-carnitine normalizes the impaired LTP and spine density in a rat model of
}

\section{global ischaemia}

Kocsis K. ${ }^{1}$, Knapp L. ${ }^{1}$, Gellért L. ${ }^{1}$, Oláh G. ${ }^{1}$, Kis Zs. ${ }^{1}$, Takakuwa H. ${ }^{3}$, Iwamori N. ${ }^{4,5}$, Ono E. ${ }^{4,5}$, Toldi J. ${ }^{1,2}$ and Farkas T. ${ }^{1}$

${ }^{1}$ Department of Physiology, Anatomy and Neuroscience, University of Szeged, Közép fasor 52, H-6726 Szeged, Hungary

${ }^{2}$ Neurology Research Group of the Hungarian Academy of Sciences and University of Szeged

${ }^{3}$ Faculty of Life Sciences, Kyoto Sangyo University, Kamigamo-motoyama, Kita, Kyoto 6038555, Japan

${ }^{4}$ Department of Biomedicine, Graduate School of Medical Sciences, Kyushu University, Fukuoka 812-8582, Japan

${ }^{5}$ Center of Biomedical Research, Research Center for Human Disease Modeling, Graduate School of Medical Sciences, Kyushu University, Fukuoka 812-8582, Japan

Corresponding author:

Tamás Farkas, Ph.D.

Institution: Department of Physiology, Anatomy and Neuroscience, University of Szeged

Address: Közép fasor 52, H-6726 Szeged, Hungary

e-mail: tfarkas@bio.u-szeged.hu

Tel.: +3662544381

Fax.: +3662544291 


\section{Abstract}

As a consequence of an ischaemic episode, the energy production is disturbed, leading to neuronal cell death. Despite intensive research, the quest for promising neuroprotective drugs has largely failed, not only because of ineffectiveness, but also because of serious side-effects and dosing difficulties. Acetyl-L-carnitine (ALC) is an essential nutrient which plays a key role in the energy metabolism by transporting fatty acids into the mitochondria for $\beta$ oxidation. It is an endogenous compound and can be used at high dose without toxicity in research into ischaemia. Its neuroprotective properties have been reported in many studies, but its potential action on long-term potentiation (LTP) and dendritic spine density has not been described to date. The aim of the present study was an evaluation of the possible protective effect of ALC after the ischaemic insult inflicted on the hippocampal synaptic plasticity in a 2-vessel occlusion (2VO) model in rat. For electrophysiological measurements, LTP was tested on hippocampal slices. The Golgi-Cox staining technique was used to determine the spine density. 2VO resulted in a decreased, unstable LTP and a significant loss of dendritic spines. ALC administered after $2 \mathrm{VO}$ was not protective, but as pretreatment prior to $2 \mathrm{VO}$ it restored the LTP nearly to the control level. This finding paralleled the histological analysis: ALC pretreatment resulted in the reappearance of dendritic spines on the CA1 pyramidal cells. Our data demonstrate that ALC administration can restore the hippocampal function and the spine density. ALC probably acts by enhancing the aerobic metabolic pathway, which is inhibited during and following the ischaemic attack.

Keywords: brain ischaemia, hippocampus, acetyl-L-carnitine, LTP, dendritic spine 


\section{Introduction}

Although ischaemic stroke is a leading cause of death (Lozano et al., 2012), there is at present hardly any effective therapy for the patients. The application of intravenous recombinant tissue plasminogen activator, which is the only neuroprotective intervention against stroke (Adams et al., 2007), is limited because victims do not meet the entry criteria of pivotal clinical trials (because of severe hypertension, an age above 80, a history of previous stroke, etc.) or they often reach the Emergency Department too late (Alderazi et al., 2012). Ischaemic stroke also results in lifelong disability. which is due to the neuronal cell death, a consequence of the excitotoxicity, the mitochondrial dysfunction and the disturbed energy production (Beal, 1992).

The quest for neuroprotective drugs for humans has largely been a failure, not only because of ineffectiveness, but also because of the severe side-effects or the dosing difficulties. Researchers have therefore started to focus on human endogenous compounds which can be administered at higher doses without toxicity.

L-Carnitine (trimethylamino- $\beta$-hydroxybutyrate), an essential nutrient in all mammalian species, exists either as free carnitine or as acylcarnitines. The acyl groups can vary considerably in length, as illustrated by the shorter acyl chain in acetyl-L-carnitine (ALC) and the longer chain in palmitoylcarnitine. The primary function of carnitine and ALC is the transportation of long- and medium-chain fatty acids into the mitochondria for $\beta$-oxidation (Virmani and Binienda, 2004). Exogenous carnitine derivatives contribute to the modulation of cholinergic neurotransmission and the nerve growth factor, to the improvement of the mitochondrial energetics and antioxidant activity, and to the modulation of protein and gene expression (reviewed by Jones et al., 2010). ALC can be synthesized in the brain by carnitineacetyltransferase (Bird et al., 1985), and can readily cross the blood-brain barrier in a 
$\mathrm{Na}^{+}$-dependent manner through the organic cation/carnitine transporter (Inano et al., 2003), which offer promising therapeutic applications.

ALC has been shown to exhibit neuroprotective effects after cerebral ischaemia in animal models, and also in cultured neurones and on brain slice preparations. ALC treatment prior to transient forebrain ischaemia prevented neuronal damage in the cortex, hippocampus and striatum of gerbils (Shuaib et al., 1995). ALC pretreatment was also found to reduce the infarct size significantly after middle cerebral artery occlusion in rats (Jalal et al., 2010). In an electrophysiological study, ALC proved to protect striatal neurones against in vitro ischaemia involving oxygen-glucose deprivation (Picconi et al., 2006).

Clinical studies have demonstrated the positive effects of ALC too. It enhanced the cerebral blood flow (Postiglione et al., 1991), and improved the energy levels, general functioning and well-being of patients (Malaguarnera et al., 2011).

The effects of ALC administration on focal in vivo and global in vivo and in vitro models have been investigated (Rosenthal et al., 1992, Shuaib et al., 1995, Picconi et al., 2006, Jalal et al., 2010, Zhang et al., 2012), but there have not yet been any reports of its effects on hippocampal long-term potentiation (LTP). We recently demonstrated a severe consequence of global hypoperfusion on the hippocampal LTP function, which can be detected by means of electrophysiological measurements and Golgi-Cox staining (Nagy et al., 2011). These methods are suitable for the investigation of the effects of potential neuroprotective agents on synaptic plasticity. We set out to determine whether ALC has a neuroprotective effect on synaptic plasticity after global hypoperfusion, and whether it influences dendritic spine genesis in the CA1 subfield of the hippocampus.

\section{Experimental procedures}

2.1 Animals and housing conditions 
Male Wistar rats weighing $220 \pm 20 \mathrm{~g}(\mathrm{~N}=45)$ supplied by Charles River Laboratories, were kept under constant environmental conditions $\left(23{ }^{\circ} \mathrm{C}\right.$; humidity $55 \pm 5 \%$; 12-h/12-h light/dark cycle) and were housed individually in standard plastic cages. Before and after all experimental procedures, all animals had free access to food and water. Every effort was made to minimize the number of animals used and their suffering. The principles of animal care (NIH publication No. 85-23), and the protocol for animal care approved both by the Hungarian Health Committee (1998) and by the European Communities Council Directive (86/609/EEC) were followed. Before the experimental procedures, all the rats were in normal health and had no neurogical deficits. The animals were randomly divided into 6 groups, as follows: sham-operated controls $(\mathrm{N}=9)$; animals that underwent 2-vessel occlusion $(2 \mathrm{VO})$ $(\mathrm{N}=10)$; a $2 \mathrm{VO}+\mathrm{ALC}$ post-treatment group $(\mathrm{N}=10)$; an ALC pretreatment+2VO group $(\mathrm{N}=10)$; an ALC pretreatment sham-operated group ( $\mathrm{N}=3)$; and a sham-operated ALC posttreatment group $(\mathrm{N}=3)$.

\subsection{Transient 2-vessel occlusion}

Transient global hypoperfusion was effected by $2 \mathrm{VO}$, described previously (Marosi et al., 2009). Anaesthesia was induced with sodium pentobarbital (60 mg/ml; i.p.), and the body temperature of the animals was maintained at $37 \pm 0.5{ }^{\circ} \mathrm{C}$ by means of an automatic heat controller (Supertech TMP-5a, Hungary). Through a midline neck incision, the common carotid arteries (CCAs) were dissected and exposed. To induce transient forebrain ischaemia, the CCAs were clamped for 30-min with non-traumatic clips (Aesculap, B. Braun Medical Ltd, Hungary). After the 30 min period, the clips were released from the CCAs and the blood flow was restarted. In the sham-operated groups, the CCAs were exposed without clamping.

\subsection{Drug administration in the experimental groups}

The effects of ALC (Sigma, Germany) on 2VO-operated and on sham-operated animals were measured in pretreatment and post-treatment. ALC was dissolved in $0.9 \%$ saline (total volume 
$1 \mathrm{ml}$ ) and was administered i.p. The animals received the treatment once a day for 5 days either before (pretreatment) or after (post-treatment) the $2 \mathrm{VO} /$ sham operation. The animals in the pretreated and pretreated sham-operated groups received the last ALC injection 1 day before the $2 \mathrm{VO}$ or sham operation. The first ALC treatment was applied $1 \mathrm{~h}$ after the surgical intervention in the post-treated and post-treated sham-operated groups.

\subsection{In vitro electrophysiology}

The animals were decapitated 5 days after the ischaemic insult. The middle parts of the hippocampi were placed in ice-cold artificial cerebrospinal fluid (aCSF) composed of (in $\mathrm{mM}$ ): $130 \mathrm{NaCl}, 3.5 \mathrm{KCl}, 1 \mathrm{NaH}_{2} \mathrm{PO}_{4}, 24 \mathrm{NaHCO}_{3}, 1 \mathrm{CaCl}_{2}, 3 \mathrm{MgSO}_{4}$ and $10 \mathrm{D}$-glucose (all from Sigma, Germany), saturated with $95 \% \mathrm{O}_{2}+5 \% \mathrm{CO}_{2}$. Coronal hippocampal slices (350 $\mu \mathrm{m})$ were prepared with a vibratome (Campden Instruments, UK), transferred to a Haas recording chamber and incubated at room temperature for $1 \mathrm{~h}$ to allow the slices to recover in the solution used for recording (differing only in that it contained $3 \mathrm{mM} \mathrm{CaCl}_{2}$ and $1.5 \mathrm{mM}$ $\mathrm{MgSO}_{4}$ ). The flow rate of the recording solution was $1.5-2 \mathrm{ml} \times \mathrm{min}^{-1}$ and the experiments were performed at a controlled chamber temperature of $34^{\circ} \mathrm{C}$.

For the electrophysiological experiments, a bipolar concentric stainless steel electrode (Neuronelektrod Ltd, Hungary) was placed in the stratum radiatum between the CA1 and CA2 regions of the hippocampal slices. The stimulus intensity was adjusted to between 30 and $60 \mu \mathrm{A}$ (constant current, $0.2-\mathrm{ms}$ pulses delivered at $0.05 \mathrm{~Hz}$ ) to evoke the half-maximum response. Field excitatory postsynaptic potentials (fEPSPs) were recorded with a 1.5-2 M $\Omega$ resistance glass micropipette filled with aCSF. The recordings were amplified with a neutralized, high input-impedance preamplifier and filtered $(1 \mathrm{~Hz}-3 \mathrm{kHz})$. The fEPSPs were digitized (AIF-03, Experimetria Ltd. Hungary), acquired at a sampling rate of $10 \mathrm{kHz}$, saved to a PC and analysed off-line with Origin 6.0 software (OriginLab Corporation, USA). The fEPSPs were monitored until the amplitudes were generally stable, and a 10-min-long 
baseline was then recorded. For LTP measurements, theta burst stimulation (TBS) was applied (bursts of 4 impulses at $100 \mathrm{~Hz}$ with a burst interval of $350 \mathrm{~ms}$ ). After the TBS, changes in fEPSP amplitudes were recorded for a further $60 \mathrm{~min}$.

\subsection{Golgi-Cox staining technique}

In order to determine the number of apical dendritic spines of the CA1 pyramidal cells, the Golgi-Cox staining method was used. The details of the preparation and staining of the hippocampal slices have been described previously (Nagy et al., 2011). The rats were decapitated and a coronal block containing the whole hippocampus was placed into GolgiCox solution and held in the dark at room temperature $\left(22-23{ }^{\circ} \mathrm{C}\right)$ for 10 days. Golgi-Cox solution is prepared from three stock solutions: a solution of 5\% potassium dichromate, a 5\% solution of potassium chromate, and a 5\% solution of mercuric chloride, all in distilled water (all from Sigma, Germany). The parts were diluted and mixed as described previously by Glaser and Van der Loos (1981). The blocks were repeatedly replaced in fresh solution every 2-3 days. After the 10-day impregnation period, the blocks were placed into $30 \%$ sucrose at 4 ${ }^{\circ} \mathrm{C}$ for at least 4 days. $100-\mu \mathrm{m}$ coronal slices from the hippocampus were cut in $6 \%$ sucrose, using a vibratome (Leica VT1000S, Leica Biosystems Nussloch GmbH, Germany). Sections were collected and mounted on gelatinized (2\%) glass slides, and were stored in a humidity chamber overnight at room temperature. The slides were placed in a glass staining tray and then processed as described by Gibb and Kolb (1998). After staining and dehydration, the slides were coverslipped with Fluoromount.

\subsection{Quantitative analysis of the dendritic spines}

The Golgi-Cox-stained hippocampal slices were studied by light microscopic stereology with the help of oil immersion objectives. The numbers of dendritic spines were measured in the control, 2VO, ALC-pretreated and ALC-post-treated groups (4 animals from each group). For the spine number analysis, a specified, $100-\mu$ m-long apical dendritic section of 15 randomly 
selected pyramidal cells was examined in the hippocampal CA1 subfield (Fig. 1). This region, which is $100-200 \mu \mathrm{m}$ from the soma, receives the most excitatory innervations from the Schaffer collaterals of the CA3 subfield (Amaral and Witter, 1989).

The apical dendrites were selected with the aid of a 100x oil-immersion lens and the images (600X) were captured through a DP70 camera connected to a light microscope (BX51, Olympus, Tokyo, Japan) and a computer. In order to achieve the whole examined area in focus, serial photomicrographs were made. The images then were stacked into one file and were studied with ImageJ 1.42q software (National Institutes of Health, USA). The numbers of spines on the $100-\mu \mathrm{m}$-long captured sections were counted visually and separately by three experimenters blind to the analysis.

\subsection{Statistical analysis}

In LTP measurements, the fEPSP amplitudes were expressed as a percentage of the 10-min baseline value before the TBS. The one-way ANOVA Tukey post hoc test was chosen for the statistical analysis of the LTP data in Fig. 2. In the course of the data analysis relating to Fig. 3, the Mann-Whitney U-test was used. For analysis of the spine density data, one-way ANOVA with the Tukey post hoc test was used (Fig. 4). SPSS10.0 for Windows software (SPSS Inc., Chicago, IL, USA) was utilized. A P value of $\leq 0.05$ was considered significant in each analysis.

\section{Results}

\subsection{In vitro electrophysiological recordings}

After recording of the 10-min, stable baseline, TBS induced in the CA2-CA1 stratum radiatum resulted in markedly increased fEPSP amplitudes in the control group (144.91 \pm $0.34 \%)$. This potentiation remained stable throughout the 1-h period of the observation. Recordings 5 days after the 30-min 2VO revealed a significant decay in LTP function, with an amplitude increase to only $118.15 \pm 0.44 \%$; it decayed over time and nearly reached the pre- 
TBS level. An fEPSP amplitude increase (134.30 $\pm 0.55 \%)$ was observed as a result of the 5day ALC post-treatment in the first $15 \mathrm{~min}$ after the TBS, but it did not reach the control level. This potentiation then slightly decayed and stabilized at $128.37 \pm 0.45 \%$. In contrast, ALC pretreatment started 5 days prior to induction of the ischaemic insult resulted in a significant improvement of the LTP function. The fESPS amplitude increase was nearly the same as in the control group (143.56 $\pm 0.35 \%)$ (Fig. 2). We also studied the incidental effects of ALC pre- or post-treatment per se on the LTP inducibility in the sham-operated control rats, but the increase in fEPSP amplitudes was unchanged by the treatment $(142.03 \pm 0.37 \%$ in the pretreated sham group and $141.39 \pm 0.37 \%$ in the post-treated sham group; data not shown). Figure 3 presents the LTP data recorded in the different experimental groups during the last 10 min of the 1-h testing following the TBS. In the control animals, TBS resulted in stable LTP, with the amplitudes remaining at $144.66 \pm 0.88 \%$ at the end of the test period. In the 2VO group, a significantly weaker LTP was induced, which displayed a marked decay over time. The potentiation level decreased to $112.20 \pm 0.86 \%$ by the end of the 1 -h period. ALC post-treatment caused much more stable LTP, which was significantly higher $(125.47 \pm$ $0.93 \%$ ) than in the $2 \mathrm{VO}$ group, but it did not regain the control level. ALC pretreatment resulted in an LTP close to the control values, and the amplitudes remained at $141.31 \pm 0.86 \%$ at the end of the recording period.

\subsection{Histology - Golgi-Cox impregnation}

Golgi-Cox staining labels a limited number of cells in a random manner. In the hippocampus, the neurones are clearly stained brown and black, and can be followed in the entire length. Fully impregnated CA1 pyramidal cells can be detected, and the spines of the apical dendrites can be studied under a light microscope. Only the density of the dendritic spines was determined in our study, even though different types of spines were always clearly seen (e.g. thin, mushroom or branched). In the control group, the spine density was $153.88 \pm 0.79 / 100$ 
$\mu \mathrm{m}$. The effect of the global hypoperfusion after a 5-day survival period was reflected in the significant decrease in the apical dendritic spine number $(70.58 \pm 1.02 / 100 \mu \mathrm{m})$. ALC posttreatment resulted in an increase in spine density $(113.63 \pm 0.73 / 100 \mu \mathrm{m})$ relative to the value for the 2VO group. A significant difference was observed between the ALC-pretreated group and the $2 \mathrm{VO}$ group, with the number of dendritic spines in the former group at almost the control level (143.75 $\pm 0.52 / 100 \mu \mathrm{m})$ (Fig. 4). These histological data point to the restoration or preservation of synaptic plasticity, which is in parallel with our electrophysiological results.

\section{Discussion}

ALC is the most widely distributed short-chain acylcarnitine in the human body (Virmani and Binienda, 2004). There are particularly high level of carnitines in the cerebellum and the hypothalamus, and lower amounts in the hippocampus and cerebral cortex (Bresolin et al., 1982, Shug et al., 1982). Despite these regional differences, the whole metabolic pool participates in fatty acid oxidation. In consequence of the strong relationship between carnitines and bioenergetic processes, these compounds have a crucial role in diseases associated with a deficient metabolism. Although glucose is the major source of energy in the central nervous system, the participation of other fuels should not be ignored during metabolic stress, such as ischaemia or neurodegenerative diseases. These substrates are ketone bodies (Robinson and Williamson, 1980), lactate (Wyss et al., 2011) and fatty acids (Ebert et al., 2003). As ALC takes part in the metabolism of the alternative energy source fatty acids, its therapeutic application could be promising.

The $2 \mathrm{VO}$ cerebral ischaemic model reduces the blood flow in the rat brain to one-third of its normal value (Farkas et al., 2007). The hippocampus, and particularly the CA1 area, is one of the brain regions most vulnerable to ischaemic insults (Pulsinelli et al., 1982). Our experiments have revealed that the damage caused by global hypoperfusion results in an 
impaired LTP function 5 days after the 2VO surgery, which was accompanied by the significant decrease in the dendritic spines of the CA1 pyramidal cells. Extensive experimental data have demonstrated the neuroprotective effect of ALC against ischaemia. Nevertheless, there has been no previous study of the potential positive effect of ALC on the impaired LTP function caused by ischaemia. Our experiments have demonstrated that ALC administration prior to the ischaemic event can be neuroprotective, resulting in a significant improvement in the LTP, the fEPSP amplitude increase after TBS proving to be the same as in the control group. In parallel with these results, histological analysis demonstrated that the number of dendritic spines returned almost to the control level.

The beneficial effect of ALC in different animal models of cerebral ischaemia has been ascribed to multiple mechanisms. One of the reported actions of this compound is protection against excitotoxicity, which appears as a result of ischaemia/reperfusion injury. Zanelli et al. demonstrated that ALC can prevent the significant cell death and can be protective in both the acute and the delayed phase of excitotoxicity. These findings can be explained by the modulation of glutamate receptors and gamma-aminobutyric acid receptors (Zanelli et al., 2005).

It has been reported that, through its antioxidant properties, ALC can reverse the decrease of the hippocampal glutathione levels after transient forebrain ischaemia to the control values (Al-Majed et al., 2006). Moreover, ALC can prevent the malondialdehyde concentration increase and improve the superoxide dismutase activity after oxygen-glucose deprivation in cell cultures (Zhang et al., 2012), and can additionally protect against lipid hydroperoxidation (Yasui et al., 2002). The antioxidant activity of ALC presumably has a role in the mechanisms underlying our findings.

Various animal experiments and clinical studies have demonstrated that disturbances in brain cholinergic transmission are associated with learning and memory dysfunctions (Yakel, 
2013). Brain ischaemia also induces an impairment of the cholinergic system: it decreases the release of ACh and eliminates the choline acetyltransferase immunoreactivity in several parts of the brain, e.g. the hippocampus (Iwasaki et al., 1996). In the hippocampal CA1 region, the LTP requires the activation of NMDA receptors. Furthermore, it has been described that cholinergic activation enhances the LTP in this subfield (Blitzer et al., 1990). ALC has been stated to exert its neuroprotective behaviour against ischaemic injury through the activation of cholinergic synaptic transmission (Ando et al., 2001, Picconi et al., 2006). The enhanced cholinergic transmission resulting from the ALC pretreatment may also serve as an explanation of the restored LTP in our experiments.

Another mechanism which possibly underlies the protective effect of ALC is the restoration of the energy status in the brain under the metabolically compromised circumstances. Molecular analysis has demonstrated that ALC treatment leads to decreases in lactate level and lactate/pyruvate ratio in the brain (Zanelli et al., 2005). The administration of ALC in supraphysiological concentration increases the intracellular level of this compound; thus, it can serve as an exogenous donor of acetyl groups and undergoes metabolization in the brain. The acetyl component of ALC enters the aerobic metabolism at a point just after the impaired reaction catalysed by pyruvate dehydrogenase. As a result, it can promote oxidative cerebral energy production and minimize anaerobic glycolysis and lactic acidosis (Zanelli et al., 2005). Previous studies indicate that ALC, by serving as an exogenous, alternative source of acetylCoA, presumably promotes oxidative cerebral energy production in our experimental system. It could preserve therefore a nearly physiological metabolism of the pyramidal cells in the hippocampal CA1 subfield and inhibited the spine density decrease, or perhaps induced the reappearance of the spines.

\section{Conclusions}


The present study provides the first report of the neuroprotective effect of ALC treatment on the LTP function impaiement caused by global hypoperfusion. It demonstrates a strict link between the LTP and dendritic spine density not only in the control, but also in the 2VO and the ALC-treated groups. In view of the literature findings, ALC can presumably exert its protective effect through different routes at the same time. These mechanisms may involve the antioxidant effect, modulation of the cholinergic transmission, protection against excitotoxicity and enhancement of the aerobic energy metabolism, whereby neurones can preserve their normal function. To clarify the exact mechanism of action of ALC on the LTP function and on the preservation or restoration of the density of dendritic spines, further experiments are needed.

\section{Acknowledgements}

This work was supported by grants from OTKA K105077 and TÁMOP 4.2.2-A-11/KONV2012-0052. This research was realized in the frames of TÁMOP 4.2.4 A/2-11-1-2012-0001 „National Excellence Program” - Elaborating and operating an inland student and researcher personal support system". The project was subsidized by the European Union and co-financed by the European Social Fund. 


\section{References}

Adams HP, Jr., del Zoppo G, Alberts MJ, Bhatt DL, Brass L, Furlan A, Grubb RL, Higashida RT, Jauch EC, Kidwell C, Lyden PD, Morgenstern LB, Qureshi AI, Rosenwasser RH, Scott PA, Wijdicks EF, American Heart Association/American Stroke Association Stroke C, American Heart Association/American Stroke Association Clinical Cardiology C, American Heart Association/American Stroke Association Cardiovascular R, Intervention C, Atherosclerotic Peripheral Vascular Disease Working G, Quality of Care Outcomes in Research Interdisciplinary Working G (2007) Guidelines for the early management of adults with ischemic stroke: a guideline from the American Heart Association/American Stroke Association Stroke Council, Clinical Cardiology Council, Cardiovascular Radiology and Intervention Council, and the Atherosclerotic Peripheral Vascular Disease and Quality of Care Outcomes in Research Interdisciplinary Working Groups: The American Academy of Neurology affirms the value of this guideline as an educational tool for neurologists. Circulation 115:e478-534.

Al-Majed AA, Sayed-Ahmed MM, Al-Omar FA, Al-Yahya AA, Aleisa AM, Al-Shabanah OA (2006) Carnitine esters prevent oxidative stress damage and energy depletion following transient forebrain ischaemia in the rat hippocampus. Clinical and experimental pharmacology \& physiology 33:725-733.

Alderazi YJ, Chang J, Yang JP, Teleb M, Chapple K, Awad A, Restrepo L (2012) Impact of Protocol Deviations in Acute Ischemic Stroke Treated With Intravenous rt-PA Within 4.5 Hours After Symptom Onset. The Neurohospitalist 2:82-86.

Amaral DG, Witter MP (1989) The three-dimensional organization of the hippocampal formation: a review of anatomical data. Neuroscience 31:571-591.

Ando S, Tadenuma T, Tanaka Y, Fukui F, Kobayashi S, Ohashi Y, Kawabata T (2001) Enhancement of learning capacity and cholinergic synaptic function by carnitine in aging rats. Journal of neuroscience research 66:266-271.

Beal MF (1992) Mechanisms of excitotoxicity in neurologic diseases. FASEB journal : official publication of the Federation of American Societies for Experimental Biology 6:3338-3344.

Bird MI, Munday LA, Saggerson ED, Clark JB (1985) Carnitine acyltransferase activities in rat brain mitochondria. Bimodal distribution, kinetic constants, regulation by malonylCoA and developmental pattern. The Biochemical journal 226:323-330.

Blitzer RD, Gil O, Landau EM (1990) Cholinergic stimulation enhances long-term potentiation in the CA1 region of rat hippocampus. Neuroscience letters 119:207-210.

Bresolin N, Freddo L, Vergani L, Angelini C (1982) Carnitine, carnitine acyltransferases, and rat brain function. Experimental neurology 78:285-292.

Ebert D, Haller RG, Walton ME (2003) Energy contribution of octanoate to intact rat brain metabolism measured by $13 \mathrm{C}$ nuclear magnetic resonance spectroscopy. The Journal of neuroscience : the official journal of the Society for Neuroscience 23:5928-5935.

Farkas E, Luiten PG, Bari F (2007) Permanent, bilateral common carotid artery occlusion in the rat: a model for chronic cerebral hypoperfusion-related neurodegenerative diseases. Brain research reviews 54:162-180.

Gibb R, Kolb B (1998) A method for vibratome sectioning of Golgi-Cox stained whole rat brain. Journal of neuroscience methods 79:1-4.

Glaser EM, Van der Loos H (1981) Analysis of thick brain sections by obverse-reverse computer microscopy: application of a new, high clarity Golgi-Nissl stain. Journal of neuroscience methods 4:117-125. 
Inano A, Sai Y, Nikaido H, Hasimoto N, Asano M, Tsuji A, Tamai I (2003) Acetyl-Lcarnitine permeability across the blood-brain barrier and involvement of carnitine transporter OCTN2. Biopharmaceutics \& drug disposition 24:357-365.

Iwasaki K, Kitamura Y, Ohgami Y, Mishima K, Fujiwara M (1996) The disruption of spatial cognition and changes in brain amino acid, monoamine and acetylcholine in rats with transient cerebral ischemia. Brain research 709:163-172.

Jalal FY, Bohlke M, Maher TJ (2010) Acetyl-L-carnitine reduces the infarct size and striatal glutamate outflow following focal cerebral ischemia in rats. Annals of the New York Academy of Sciences 1199:95-104.

Jones LL, McDonald DA, Borum PR (2010) Acylcarnitines: role in brain. Progress in lipid research 49:61-75.

Lozano R, Naghavi M, Foreman K, Lim S, Shibuya K, Aboyans V, Abraham J, Adair T, Aggarwal R, Ahn SY, Alvarado M, Anderson HR, Anderson LM, Andrews KG, Atkinson C, Baddour LM, Barker-Collo S, Bartels DH, Bell ML, Benjamin EJ, Bennett D, Bhalla K, Bikbov B, Bin Abdulhak A, Birbeck G, Blyth F, Bolliger I, Boufous S, Bucello C, Burch M, Burney P, Carapetis J, Chen H, Chou D, Chugh SS, Coffeng LE, Colan SD, Colquhoun S, Colson KE, Condon J, Connor MD, Cooper LT, Corriere M, Cortinovis M, de Vaccaro KC, Couser W, Cowie BC, Criqui MH, Cross M, Dabhadkar KC, Dahodwala N, De Leo D, Degenhardt L, Delossantos A, Denenberg J, Des Jarlais DC, Dharmaratne SD, Dorsey ER, Driscoll T, Duber H, Ebel B, Erwin PJ, Espindola P, Ezzati M, Feigin V, Flaxman AD, Forouzanfar MH, Fowkes FG, Franklin R, Fransen M, Freeman MK, Gabriel SE, Gakidou E, Gaspari F, Gillum RF, Gonzalez-Medina D, Halasa YA, Haring D, Harrison JE, Havmoeller R, Hay RJ, Hoen B, Hotez PJ, Hoy D, Jacobsen KH, James SL, Jasrasaria R, Jayaraman S, Johns N, Karthikeyan G, Kassebaum N, Keren A, Khoo JP, Knowlton LM, Kobusingye O, Koranteng A, Krishnamurthi R, Lipnick M, Lipshultz SE, Ohno SL, Mabweijano J, MacIntyre MF, Mallinger L, March L, Marks GB, Marks R, Matsumori A, Matzopoulos R, Mayosi BM, McAnulty JH, McDermott MM, McGrath J, Mensah GA, Merriman TR, Michaud C, Miller M, Miller TR, Mock C, Mocumbi AO, Mokdad AA, Moran A, Mulholland K, Nair MN, Naldi L, Narayan KM, Nasseri K, Norman P, O'Donnell M, Omer SB, Ortblad K, Osborne R, Ozgediz D, Pahari B, Pandian JD, Rivero AP, Padilla RP, Perez-Ruiz F, Perico N, Phillips D, Pierce K, Pope CA, 3rd, Porrini E, Pourmalek F, Raju M, Ranganathan D, Rehm JT, Rein DB, Remuzzi G, Rivara FP, Roberts T, De Leon FR, Rosenfeld LC, Rushton L, Sacco RL, Salomon JA, Sampson U, Sanman E, Schwebel DC, Segui-Gomez M, Shepard DS, Singh D, Singleton J, Sliwa K, Smith E, Steer A, Taylor JA, Thomas B, Tleyjeh IM, Towbin JA, Truelsen T, Undurraga EA, Venketasubramanian N, Vijayakumar L, Vos T, Wagner GR, Wang M, Wang W, Watt K, Weinstock MA, Weintraub R, Wilkinson JD, Woolf AD, Wulf S, Yeh PH, Yip P, Zabetian A, Zheng ZJ, Lopez AD, Murray CJ, AlMazroa MA, Memish ZA (2012) Global and regional mortality from 235 causes of death for 20 age groups in 1990 and 2010: a systematic analysis for the Global Burden of Disease Study 2010. Lancet 380:2095-2128.

Malaguarnera M, Bella R, Vacante M, Giordano M, Malaguarnera G, Gargante MP, Motta M, Mistretta A, Rampello L, Pennisi G (2011) Acetyl-L-carnitine reduces depression and improves quality of life in patients with minimal hepatic encephalopathy. Scandinavian journal of gastroenterology 46:750-759.

Marosi M, Fuzik J, Nagy D, Rakos G, Kis Z, Vecsei L, Toldi J, Ruban-Matuzani A, Teichberg VI, Farkas T (2009) Oxaloacetate restores the long-term potentiation impaired in rat hippocampus CA1 region by 2-vessel occlusion. European journal of pharmacology 604:51-57. 
Nagy D, Kocsis K, Fuzik J, Marosi M, Kis Z, Teichberg VI, Toldi J, Farkas T (2011) Kainate postconditioning restores LTP in ischemic hippocampal CA1: onset-dependent second pathophysiological stress. Neuropharmacology 61:1026-1032.

Picconi B, Barone I, Pisani A, Nicolai R, Benatti P, Bernardi G, Calvani M, Calabresi P (2006) Acetyl-L-carnitine protects striatal neurons against in vitro ischemia: the role of endogenous acetylcholine. Neuropharmacology 50:917-923.

Postiglione A, Soricelli A, Cicerano U, Mansi L, De Chiara S, Gallotta G, Schettini G, Salvatore M (1991) Effect of acute administration of L-acetyl carnitine on cerebral blood flow in patients with chronic cerebral infarct. Pharmacological research : the official journal of the Italian Pharmacological Society 23:241-246.

Pulsinelli WA, Brierley JB, Plum F (1982) Temporal profile of neuronal damage in a model of transient forebrain ischemia. Annals of neurology 11:491-498.

Robinson AM, Williamson DH (1980) Physiological roles of ketone bodies as substrates and signals in mammalian tissues. Physiological reviews 60:143-187.

Rosenthal RE, Williams R, Bogaert YE, Getson PR, Fiskum G (1992) Prevention of postischemic canine neurological injury through potentiation of brain energy metabolism by acetyl-L-carnitine. Stroke; a journal of cerebral circulation 23:13121317; discussion 1317-1318.

Shuaib A, Waqaar T, Wishart T, Kanthan R, Howlett W (1995) Acetyl-L-carnitine attenuates neuronal damage in gerbils with transient forebrain ischemia only when given before the insult. Neurochemical research 20:1021-1025.

Shug AL, Schmidt MJ, Golden GT, Fariello RG (1982) The distribution and role of carnitine in the mammalian brain. Life sciences 31:2869-2874.

Virmani A, Binienda Z (2004) Role of carnitine esters in brain neuropathology. Molecular aspects of medicine 25:533-549.

Wyss MT, Jolivet R, Buck A, Magistretti PJ, Weber B (2011) In vivo evidence for lactate as a neuronal energy source. The Journal of neuroscience : the official journal of the Society for Neuroscience 31:7477-7485.

Yakel JL (2013) Cholinergic receptors: functional role of nicotinic ACh receptors in brain circuits and disease. Pflugers Archiv : European journal of physiology 465:441-450.

Yasui F, Matsugo S, Ishibashi M, Kajita T, Ezashi Y, Oomura Y, Kojo S, Sasaki K (2002) Effects of chronic acetyl-L-carnitine treatment on brain lipid hydroperoxide level and passive avoidance learning in senescence-accelerated mice. Neuroscience letters 334:177-180.

Zanelli SA, Solenski NJ, Rosenthal RE, Fiskum G (2005) Mechanisms of ischemic neuroprotection by acetyl-L-carnitine. Annals of the New York Academy of Sciences 1053:153-161.

Zhang R, Zhang H, Zhang Z, Wang T, Niu J, Cui D, Xu S (2012) Neuroprotective Effects of Pre-Treament with 1-Carnitine and Acetyl-1-Carnitine on Ischemic Injury In Vivo and In Vitro. International journal of molecular sciences 13:2078-2090. 
Fig. 1: Illustration of the measured region on hippocampal slices after Golgi-Cox impregnation. The first photomicrograph reveals a coronal segment of the hippocampus and a representative pyramidal cell (enlarged section), with a small rectangle showing the examined apical dendritic region. The photomicrographs of the control, 2VO, ALC-post-treated and ALC-pretreated groups depict the site of spine density measurements. Note the fewer dendritic spines in the $2 \mathrm{VO}$ group relative either to the control or to the ALC-pretreated group. Scale bar: $500 \mu \mathrm{m}, 100 \mu \mathrm{m}$ and $10 \mu \mathrm{m}$, respectively.

Fig. 2: Changes in fEPSP amplitudes after TBS (number of electrophysiological recordings: $\mathrm{N}=9$ from the control, $\mathrm{N}=8$ from the $2 \mathrm{VO} 5 \mathrm{~d}, \mathrm{~N}=9$ from the ALC post-treatment and $\mathrm{N}=9$ from the ALC pretreatment group). The global hypoperfusion resulted in significantly decreased fEPSP amplitudes after TBS relative to the control values $(* * \mathrm{P}<0.01$ for the period 11-17 $\mathrm{min}, * * * \mathrm{P}<0.001$ after $18 \mathrm{~min}$ until the end of the recording period). ALC administered for 5 days after $2 \mathrm{VO}$ did not completely prevent this decay. However, the data from this group were significantly different from those from the $2 \mathrm{VO}$ group from $30 \mathrm{~min}$ after the TBS $\left({ }^{\#} \mathrm{P}<0.05\right.$ for the period $29-40 \mathrm{~min},{ }^{\# \#} \mathrm{P}<0.01$ for the period $41-49 \mathrm{~min},{ }^{\# \# \#} \mathrm{P}<0.001$ after 50 min), and the LTP function also differed markedly from that in the control group $(* * \mathrm{P}<0.01$ for the period 24-37 min). ALC was protective against the ischaemic injury when it was administered for 5 days prior to the $2 \mathrm{VO}$ surgery. The increase in fESPS amplitudes was nearly as same as in the control group; the data did not show a significant difference between the two groups at any time point. The LTP in the pretreated group was significantly stronger and more stable than that in the 2 VO group $\left({ }^{\# \#} \mathrm{P}<0.001\right.$ from 20 min after the TBS until the end of the recording period). Data points are means \pm S.E.M. of normalized amplitudes of fEPSPs. Asterisks denote significant differences from the control, and ${ }^{\#}$ denotes significant differences from the data for the 2 VO group $\left(* * \mathrm{P}<0.01, * * * \mathrm{P}<0.001,{ }^{\#} \mathrm{P}<0.05,{ }^{\# \#} \mathrm{P}<0.01\right.$,

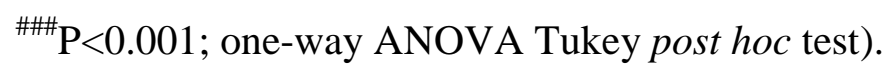


Fig. 3: The level of the LTP at the end of the recordings. The columns demonstrate the potentiation of fEPSP amplitudes recorded during the last $10 \mathrm{~min}$ (between 50 and $60 \mathrm{~min}$ ) of the 1-h testing following the TBS. The marked LTP induced in the control group remained stable at the end of the measurements. The fEPSPs displayed decaying amplitudes over time in the $2 \mathrm{VO}$ group, and nearly reached the pre-TBS level in the last $10 \mathrm{~min}$. Although a higher LTP was induced in the ALC post-treatment group, it also later stabilized at a lower level. ALC administration for 5 days before the ischaemic insult proved protective, since a significantly higher LTP was induced in this group relative to the $2 \mathrm{VO}$ group. The level of this potentiation was close to that for the control and remained stable throughout the recording period. Data points are means \pm S.E.M. of normalized amplitudes of fEPSPs in the last 10 min. Asterisks denote significant differences from the control, and ${ }^{\#}$ denotes significant differences from the 2VO group $\left(* \mathrm{P}<0.05, * * * \mathrm{P}<0.001,{ }^{\# \# \#} \mathrm{P}<0.001\right.$; Mann-Whitney U-test).

Fig. 4: Changes in spine density in the Golgi-Cox-stained hippocampal CA1 regions. 15 apical dendritic sections were measured from each of 4 animals in each group. The analysis revealed that transient global hypoperfusion induced a significant reduction in spine density 5 days after 2VO. ALC post-treatment caused a definitive increase in dendritic spine number relative to the $2 \mathrm{VO}$ group, but the number did not reach the control level. The protective effect of ALC pretreatment was revealed by histological analysis. As a result of ALC pretreatment, the dendritic spine density was found to be close to the control level. The histogram shows means \pm S.E.M. of the dendritic spine numbers. There are significant differences between all of the examined groups at the $\mathrm{P}<0.001$ level (one-way ANOVA, Tukey post hoc test). 

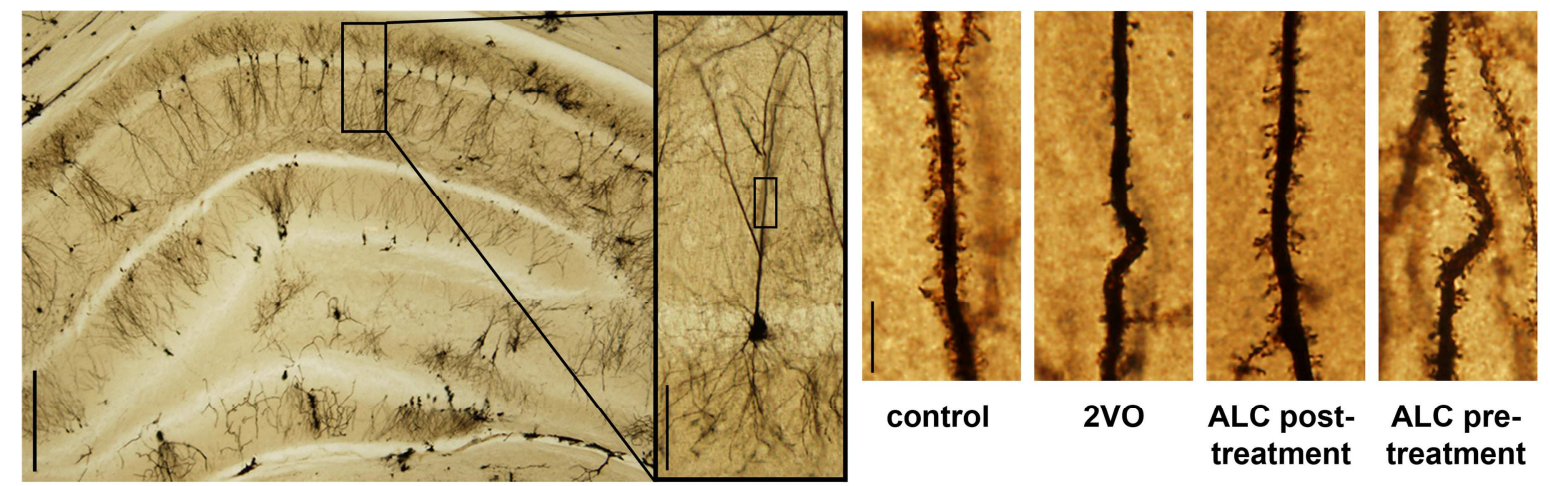

control

2Vo

ALC post- ALC pretreatment treatment

Fig. 1 


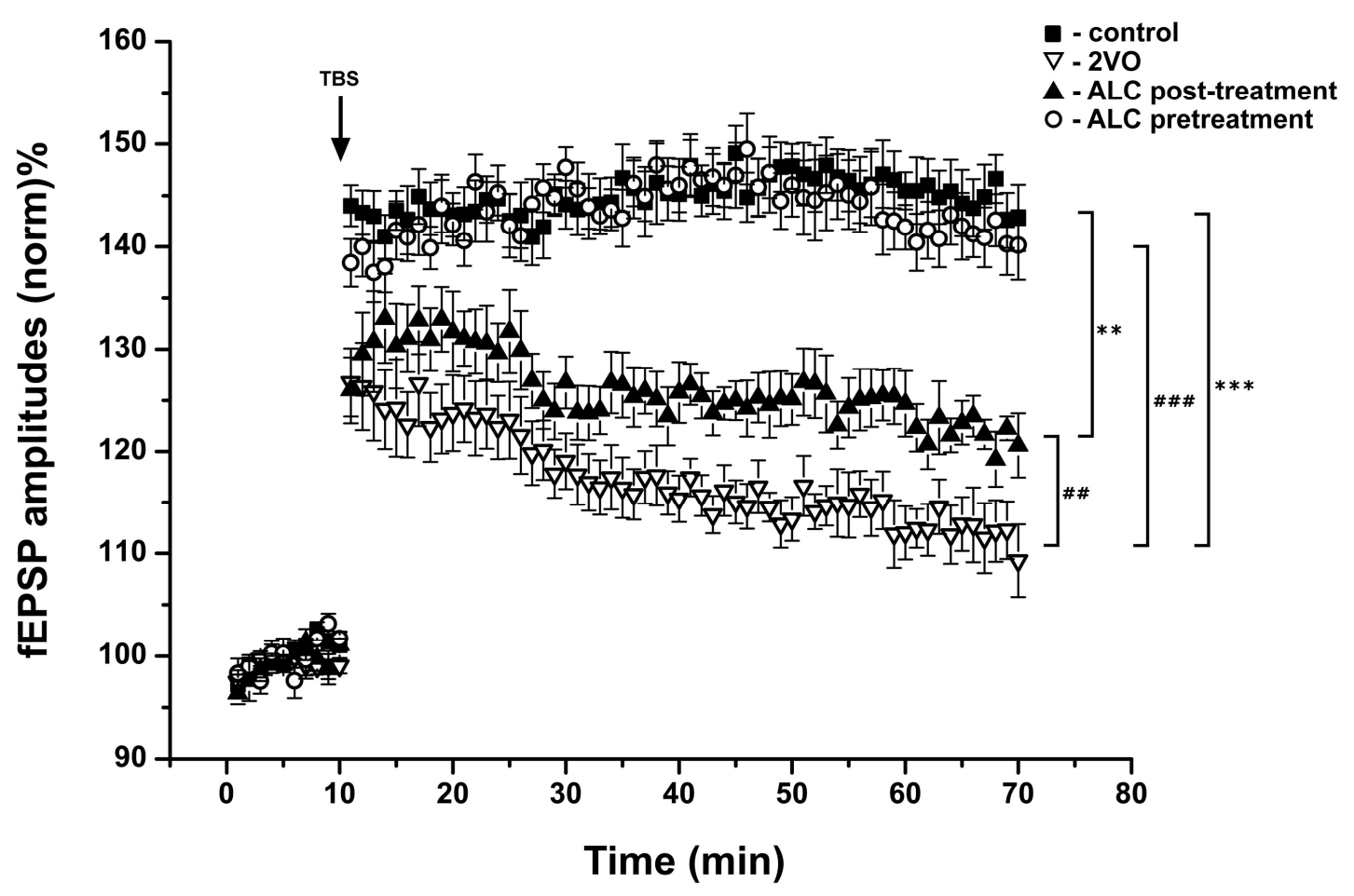

Fig. 2 


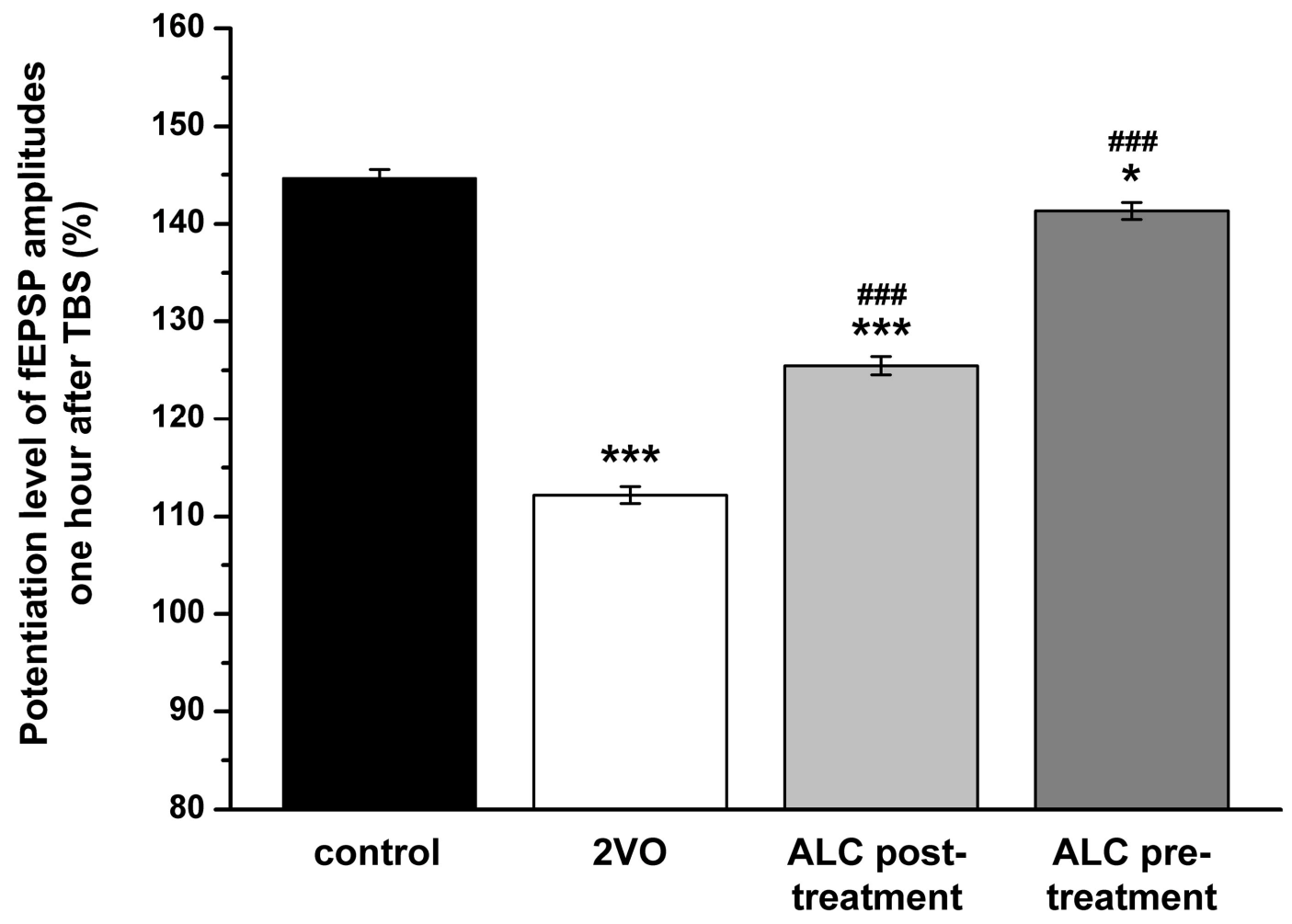

Fig. 3 


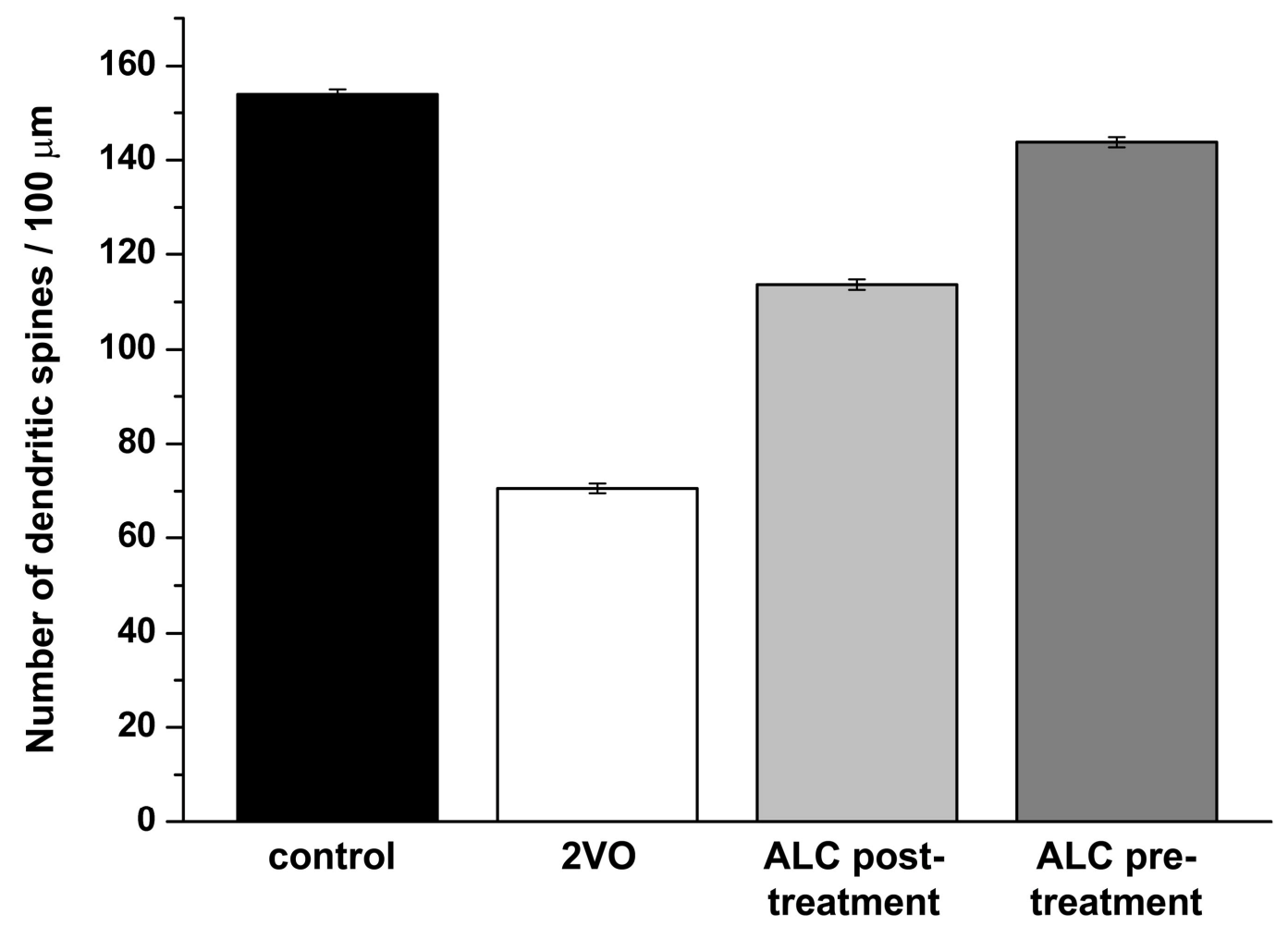

Fig. 4 